\title{
陶瓷固化体的浸出行为及其机理
}

\author{
孙亚平，王洪龙，褚 健，王 绪，潘社奇，张 铭 \\ (中国工程物理研究院 材料研究所, 江油 621908)
}

摘 要: 高放废物(HLW)在深地质处置后, 其中的放射性核素有可能浸出并伴随地下水循环进入人类环境。这是固 化体中放射性核素进入生物圈最可能的途径, 因此 HLW 固化体的化学稳定性是固化基材篮选的主要依据。陶瓷固 化体作为第二代 HLW 固化体，具有长程有序的特点，相比玻璃固化体，更容易定量表征，这对于固化体浸出机理 的研究有着重要的意义。然而陶瓷固化体的浸出机理与评价方法研究都处于起步阶段, 也缺乏被处置库接收的标 准。为规范/建全陶瓷固化体化学稳定性评价方法, 认识放射性核素的浸出机制, 本文概述了核废物固化体化学稳 定性研究方法、研究重点; 总结了相关陶瓷的水热蚀变研究现状, 分析了其中核素的浸出率; 探讨了影响因素及其 影响方式; 最后归纳了目前提出的浸出机制以及存在的问题。

关 键 词: 化学稳定性; 固化体; 浸出机制; 综述

中图分类号: TL94 文献标识码: A

\section{Leaching Behavior and Mechanism of Ceramic Waste Forms}

\author{
SUN Ya-Ping, WANG Hong-Long, CHU Jian, WANG Xu, PAN She-Qi, ZHANG Ming \\ (Institute of Materials, China Academy of Engineering Physics, Jiangyou 621908, China)
}

\begin{abstract}
The radionuclides in high-level radioactive waste (HLW) forms may be leached out over geological time and enter the human environment with groundwater circulation after deep geological disposal. This is likely the potential way for radionuclides to enter the biosphere. Therefore, the long-term chemical durability of HLW waste forms is the key issue for selecting suitable waste forms. Ceramic waste forms, regarded as the second generation of HLW forms, are of long-range ordered structures, whose physical properties can be easily characterized quantitatively. It is of great importance and significance to study the leaching mechanism of ceramic waste forms. However, not only the studies of leach mechanisms, but also the evaluation methods of ceramic waste forms are in their infancy. There is also lack of the standards of ceramic waste forms received by the repository. The present paper summarizes the research methods and key points of chemical durability, reviews the research status of hydrothermal alteration of related ceramics, and analyzes the leaching rate of radionuclides. In addition, the paper also discusses the influencing factors and their influencing modes, and finally concludes the leaching mechanisms and existing problems.
\end{abstract}

Key words: chemical durability; waste form; leaching mechanism; review

核电站运行、乏燃料处理以及其它核技术的应 用会产生放射性废物。高放废物(High-radioactive
Level Waste, HLW)中含有大量中长寿命的超铀核素 和裂变产物, 需要很多年的衰变才能达到无害化水

收稿日期：2018-08-15; 收到修改稿日期：2018-11-29

基金项目：中国博士后基金(2018M633410); 国家自然科学基金(41372055)

Chinese Postdoctoral Science Foundation (2018M633410); National Natural Science Foundation of China (41372055)

作者简介：孙亚平(1987-), 女，博士. E-mail: ypsun0717@163.com

通讯作者: 张 铭, 教授. E-mail: mz10001_mzhang@sina.com 
平。HLW 的处理处置是世界性难题, 目前大多数国 家的 HLW 没有得到最终处置, 还在等待更加安全 并能被普遍接受的处理处置技术的出现。国际上公 认的最安全可行的 HLW 处置方式是深地质处置 ${ }^{[1-2]}$, 在处置之前需要将 HLW 固化成密实、机械强度高、 化学惰性的固体形态。因此, 固化体是防止放射性 核素进入生物圈的第一层屏障。如果固化体在极端 条件下(如破碎、地下水渗入等)能包容和固定 HLW, 将大大降低处置库的设计要求, 增加公众对 HLW 深地质处置的信心。

HLW 固化方法有煅烧固化、玻璃固化、陶瓷固 化、玻璃陶瓷固化、人造岩石固化(也是一种陶瓷, 后文放在陶瓷中讨论)等。目前只有玻璃固化在一些 发达国家得到了工业化应用, 其它方法还处于研究 阶段。玻璃固化将放射性核素包容在玻璃三维网络 中，优点是能同时固化多种放射性核素，且工艺简 单; 缺点是属于亚稳态物质, 在数百度温度或潮湿 等地质条件下，抗浸出性等性能下降 ${ }^{[3]}$ 。此外，构成 玻璃网络形成体(Form)的 $\mathrm{FO}_{4}$ 四面体 $(\mathrm{F}=\mathrm{Si} 、 \mathrm{~B} 、 \mathrm{Zr}$ 、 $\mathrm{Al} 、 \mathrm{P})$ 是长程无序的, 较难进行微观结构的定量研 究 ${ }^{[4]}$ 。陶瓷固化利用“类质同象替代”原理将 HLW 固 定在陶瓷的晶格中, 因此陶瓷固化体的化学、热、 机械以及辐照稳定性更好, 特别适合钣系核素的固 化, 是第二代 HLW 固化体 ${ }^{[5-6]}$; 同时陶瓷的结构具 有对称性和周期性, 有序度高, 是研究固化体浸出 行为和机理的较好选择, 但是陶瓷对废物的选择性 强, 生产工艺复杂, 生产成本高。表 1 为陶瓷固化和 玻璃固化的比较 ${ }^{[7]}$ 。

目前适合 HLW 固化的陶瓷有十多种: 锆石、榍 石、磷灰石、独居士、磷钎矿、烧绿石、斜锆石、 钲钛矿等, 如表 2 所示 ${ }^{[8]}$ 。这些陶瓷硬度大、不易解 理、不易破碎, 在地球上都有对应的稳定矿物。除 榍石基玻璃陶瓷在加拿大获得了实际应用外 ${ }^{[9]}$, 其 它都处于研究阶段。

处置一段时间后, 处置库的工程屏障可能失效 (如 1000 年), 届时地下水有机会直接接触 HLW 固 化体，使其中的放射性核素或其他有害元素溶出,

表 1 玻璃固化和陶瓷固化的优缺点比较 ${ }^{[7]}$

Table 1 Advantages and disadvantages of glass immobilization and ceramic immobilization ${ }^{[7]}$

\begin{tabular}{ccc}
\hline Parameters & Glass & Ceramic \\
\hline Loading of waste $/ \mathrm{wt} \%$ & $10-30$ & $15-30$ \\
Density $/\left(\mathrm{g} \cdot \mathrm{cm}^{-3}\right)$ & $2.5-2.8$ & $3.0-5.8$ \\
Leach rate $/\left(\mathrm{g} \cdot \mathrm{cm}^{-2} \cdot \mathrm{d}^{-1}\right)$ & $10^{-4}-10^{-7}$ & $10^{-6}-10^{-10}$ \\
Anti-pressure ability & Low & High \\
Radiation tolerance $/ \mathrm{Gy}$ & $10^{-9}$ & $\sim 10^{-9}$ \\
\hline
\end{tabular}

表 2 陶瓷固化体的主要矿相 ${ }^{[8]}$

Table 2 Main mineral of ceramic waste forms ${ }^{[8]}$

\begin{tabular}{ccc}
\hline Mineral & Formula & Immobilized nuclide $^{\mathrm{a}}$ \\
\hline Zircon & $\mathrm{ZrSiO}_{4}$ & $\mathrm{An}$ \\
Titanite & $\mathrm{CaTiSiO}_{5}$ & $\mathrm{Ln}, \mathrm{An}$ \\
Apatite & $\mathrm{Ca}_{5}\left(\mathrm{PO}_{4}\right)_{3}(\mathrm{OH}, \mathrm{F}, \mathrm{O})$ & $\mathrm{U}, \mathrm{Th}, \mathrm{REE}, \mathrm{I}, \mathrm{Cs}$ \\
Monazite & $\mathrm{CePO}_{4}$ & $\mathrm{Ce}, \mathrm{La}, \mathrm{Eu}, \mathrm{Gd}, \mathrm{U}, \mathrm{LREE}$ \\
Xenotime & $\mathrm{YPO}_{4}$ & $\mathrm{HREE}$ \\
Pyrochlore & $\mathrm{CaUTi}_{2} \mathrm{O}_{7}$ & $\mathrm{Ln}, \mathrm{An}$ \\
Baddeleyite & $\mathrm{ZrO}_{2}$ & $\mathrm{Ln}, \mathrm{An}$ \\
Perovskite & $\mathrm{CaTiO}_{3}$ & $\mathrm{Sr}, \mathrm{REE}, \mathrm{Fe}, \mathrm{Na}, \mathrm{An}$ \\
Zirconolite & $\mathrm{CaZrTi}_{2} \mathrm{O}_{7}$ & $\mathrm{Ln}, \mathrm{An}, \mathrm{Fe}, \mathrm{Ni}, \mathrm{Cr}, \mathrm{Zr}$ \\
Brannerite & $\mathrm{UTi}_{2} \mathrm{O}_{6}$ & $\mathrm{Ln}, \mathrm{An}$ \\
Rutile & $\mathrm{TiO}_{2}$ & $\mathrm{Zr}$ \\
Alkali & $\mathrm{BaAl}_{2} \mathrm{Ti}_{6} \mathrm{O}_{16}$ & $\mathrm{Cs}, \mathrm{Sr}, \mathrm{Ba}, \mathrm{Rb}, \mathrm{A} 1$ \\
Psilomelane & &
\end{tabular}

a: An, Ln, REE represent actinides, lanthanides, and rare earth elements, respectively.

并通过地下水循环带入人类环境。这是固化体中放 射性核素进入生物圈的最可能途径，是决定深地质 处置是否可行的关键因素 ${ }^{[10]}$ 。因此, 固化体的化学 稳定性能是固化体篎选的主要依据, 也是地质处置 安全论证的必要环节。但是 HLW 地质处置时间长, 难以在实验室塑造相似的地质条件进行长达 10 万 年的研究。同时, 固化体成分复杂, 难以使用单一模 型或理论解释其中核素的浸出行为。上世纪七十年 代以来, 国内外对很多固化体的化学稳定性、地质 处置安全性和对环境的影响进行了研究 ${ }^{[11]}$, 包括实 验室研究、地下实验室现场试验、自然类比论证以 及数学模型推算等。建立了静态浸出、动态浸出等 一系列的实验方法，提出了玻璃固化体和废物包特 性鉴定与接受准则。但是固化体的化学稳定性、浸 出行为和机理的研究依然很浅显, 缺乏系统性, 尤 其是陶瓷固化缺乏实践经验以及被处置库接收的标 准。本文总结了 HLW 陶瓷固化体化学稳定性的研 究方法、研究现状、存在的问题, 希望为固化体的 评价和评价方法的建立提供参考。

\section{1 化学稳定性研究方法}

固化体在地下水浸泡下的长期稳定性是评估固 化体安全的重要依据，合格的固化体应该具有抗 水、碱、酸、盐、气体侵蚀的能力。目前运用较多的 实验标准有 $\mathrm{MCC}$ (Materials Characterization Center) ${ }^{[12]}$ 和 PCT (Standard Product Consistency Test) ${ }^{[13]}$ 。表 3 列出了 MCC 和 PCT 实验标准及其应用范围。 MCC 是使用块状样品进行实验, 这与实际处置在地下的 
表 3 核废物固化体的浸出实验标准

Table 3 Standard leaching test methods for nuclear waste forms

\begin{tabular}{lcccc}
\hline Sample & State & $\begin{array}{c}\text { Temperature/ } \\
{ }^{\circ} \mathrm{C}\end{array}$ & $\begin{array}{c}(S A / V) / \\
\left(\mathrm{m}^{-1} \cdot \mathrm{g}^{-1}\right)\end{array}$ & $\begin{array}{c}\text { Duration } \\
\text { time } / \mathrm{d}\end{array}$ \\
\hline MCC-1 & Static & $40,70,90$ & 10 & $3,7,14,28$ \\
MCC-2 & Static & $150,200,250$ & 10 & $3,7,14,28$ \\
MCC-3 & Static & 90,150 & 680 & \\
MCC-4 & Dynamic & 75 & & \\
PCT-A & Static & 90 & 1000 & 7 \\
PCT-B & Static & 90 & $1000^{\mathrm{b}}$ & 28 \\
PCT-C & Static & $40,70,90$ & $1000^{\mathrm{b}}$ & 28 \\
PCT-D & Static & 90 & $1000^{\mathrm{b}}$ & $56,182,364 \ldots$ \\
PCT-E & Static & $40,70,90$ & $1000^{\mathrm{b}}$ & $56,182,364 \ldots$ \\
\hline
\end{tabular}

b: Reference value

固化体形态一致, 优点是实验数据重复性高, 缺点 是实验周期长。 $\mathrm{PCT}$ 是通过增大固-液接触面积(减 小固化体表面积与浸出剂体积比 $(S A / V)$ 值)来加速实 验, 属于快速浸出法。对样品量很少的特殊样品也 可进行试验, 但实验结果重现性差, 样品表面分析 困难。在固化体的抗浸出实验中, 动态浸出法还考 虑了地下水的流速。

这些方法研究了环境温度、浸出液成分、浸出 液 $\mathrm{pH} 、 S A / V$ 、固化体包容物以及包容量等对化学稳 定性的影响。除表 3 所列的方法外, 还有根据 Soxhlet 提取原理进行改进的动态浸出法 MCC-5; 更接近地质环境水蒸气情形的蒸气浸出法; 研究热 力学稳定性以及脆性的 MCC-10; 研究拉伸强度的 MCC-11; 研究放射稳定性的 MCC-6、MCC-12 ${ }^{[14]}$ 。

\section{2 实验研究对象}

固化体中放射性核素的浸出行为一般用浸出 率、溶解速率、水化层和第二相等来表征。

\section{1 浸出率}

固化体的化学稳定性一般用抗浸出性能来表示, 元素浸出率越低, 化学稳定性越好。归一化浸出率 使用(1)式计算 ${ }^{[13]}$ :

$$
N L_{\mathrm{i}}=c_{\mathrm{i}}\left[\left[(S A / V) \cdot f_{\mathrm{i}}\right]\right.
$$

$N L_{\mathrm{i}}$ 为元素 $\mathrm{i}$ 归一化浸出率, $\mathrm{g} / \mathrm{m}^{2} ; c_{\mathrm{i}}$ 为浸出液中元素 $\mathrm{i}$ 的浓度, $\mathrm{g} / \mathrm{L} ; S A$ 为浸出液中固化体总表面积, $\mathrm{m}^{2} ; V$ 为浸出液体积, $\mathrm{L} ; f_{\mathrm{i}}$ 为固化体中 $\mathrm{i}$ 元素的质量分数。 我国行标 EJ 1186-2005《放射性废物体和废物包的 特性鉴定》 ${ }^{[15]}$ 对玻璃固化体的要求是：在样品表面 积/浸出剂体积之比为 $(10.0 \pm 0.5) / \mathrm{m}$ 的条件下, 在 $(90 \pm 1){ }^{\circ} \mathrm{C}$ 去离子水中, 静态浸出 $28 \mathrm{~d}$ 的单位表面积 总失重应小于 $15 \mathrm{~g} / \mathrm{m}^{2}, \mathrm{Si} 、 \mathrm{~B} 、 \mathrm{Na}$ 和 $\mathrm{Cs} 、 \mathrm{U}$ 的归一
化元素浸出率应低于 $1 \mathrm{~g} /\left(\mathrm{m}^{2} \cdot \mathrm{d}\right)$ 。一般而言, 硼硅酸 盐玻璃固化体中钶系元素的浸出率为 $0.1 \mathrm{~g} /\left(\mathrm{m}^{2} \cdot \mathrm{d}\right)$, 改良后可低至 $0.01 \mathrm{~g} /\left(\mathrm{m}^{2} \cdot \mathrm{d}\right)^{[3]}$ 。陶瓷固化体被处置库 接收的标准还没有制定, 但是由表 1 可知, 陶瓷固 化体的浸出率比玻璃低 2 3 个数量级。

\section{2 溶解速率}

溶解速率由固体中浸出的离子浓度计算而得, 如矿物(Mineral)的溶解速率可表示为 ${ }^{[16]}$.

$$
R_{\min , \mathrm{i}}=\left(C_{\mathrm{i}} \cdot F\right) /\left(v_{\min , \mathrm{i}} \cdot S\right)
$$

$R_{\min , \mathrm{i}}$ 为基于 $\mathrm{i}$ 元素浸出率计算的溶解速率, $\mathrm{mol} /$ $\left(\mathrm{cm}^{2} \cdot \mathrm{s}\right) ; C_{\mathrm{i}}$ 为浸出液中 $\mathrm{i}$ 元素浓度, $\mathrm{mol} / \mathrm{kg} ; F$ 代表开 放体系液体流动速率, $\mathrm{kg} / \mathrm{s} ; v_{\min , \mathrm{i}}$ 表示矿物中 $\mathrm{i}$ 元素 的摩尔分数; $S$ 表示反应釜中此矿物总的 BET 表面 积, $\mathrm{cm}^{2}$ 。一般选择不易沉淀、容易检测、检出限低 的离子作为表征。Icenhower 等 ${ }^{[17]}$ 使用 $\mathrm{Gd}$ 表征钲钛 锆石和钛铀矿的溶解速率; Kohler 等 ${ }^{[16]}$ 采用 $\mathrm{Ca}$ 表征 天然磷灰石的溶解速率。近年来较少使用溶解速率 表征固化体的化学稳定性, 主要是因为式(2)中的 $C_{\mathrm{i}}$ 并不一定全是来自溶解，也可能有离子交换、扩散 等的贡献。但是它对后文溶解一沉淀浸出模型的理解 至关重要。

\section{3 水化层和第二相}

固化体浸泡一段时间后，其中的放射性核素会 浸出，溶液中的水会浸入，因此在表面形成一个较 为疏松的水化层/胶体层。对于玻璃固化体而言，水 化层通常抑制放射性核素的浸出，起“保护”固化体 的作用，但对陶瓷固化体的研究较少。第二相的形 成与固化体的成分直接相关，如过渡金属、REE、 钣系等高价金属容易发生水化反应，浸出到固化体 表面; 当其在溶液中达到饱和浓度时，将发生沉淀、 絮凝，附着在水化层或者形成第二相。通过对水化 层和第二相的研究可了解固化体中元素的迁移模式, 再结合浸出液的分析，可推断其浸出机制。表 4 列

表 4 陶瓷水热蚀变水化层和第二相

Table 4 The reaction layer and second phase upon

\begin{tabular}{|c|c|c|c|c|}
\hline \multirow{2}{*}{ Ceramic } & \multicolumn{2}{|c|}{ Hydration layer } & \multicolumn{2}{|c|}{ Second phase } \\
\hline & Thickness & Method & Constituent & Method \\
\hline Titanite $^{[18]}$ & $100 \mathrm{~nm} \sim$ & SIMS & $\mathrm{TiO}_{2}$, etc & EDX \\
\hline $\operatorname{Zicon}^{[19]}$ & $\sim 30 \mu \mathrm{m} \sim$ & EMP & $\mathrm{m} / \mathrm{t}-\mathrm{ZrO}_{2}$ & EMP \\
\hline Zirconolite $^{[20]}$ & $1-90 \mathrm{~nm}$ & Calcalation $^{\mathrm{c}}$ & Ti-, $\mathrm{Zr}(\mathrm{OH})_{4}$ & ICP-MS \\
\hline Monazite $^{[21]}$ & (Sub) nm & BSE & Rhabdophane & Raman \\
\hline Pyrochlore $^{[22]}$ & & & $\begin{array}{c}\text { Brannerite, } \\
\text { rutile }\end{array}$ & XRD \\
\hline Apatite $^{[16]}$ & & & $\mathrm{APO}_{4}$ & \\
\hline
\end{tabular}
ceramics after hydrothermal alteration

c: The sample thicknesses were equivalent covered from concentrations of cations released into solution 
出了陶瓷水热蚀变后的表面水化层、第二相, 及其 相对应的分析方法, 由表可见, 这些方法基本属于 半定量方法，亟待采用新型的分析方法。

\section{3 浸出行为影响因素}

固化体中放射性核素的浸出受很多因素的影响, 可分为固化体本身因素(如相组成、致密度等 $)^{[23]}$, 所含 HLW 的因素(如组成、活度、价态、水合/配位 能力等), 环境条件(温度、地下水成分、流速、 $\mathrm{pH}$ 、 微生物等 ${ }^{[24]}$ 三类。在不同环境条件和不同反应阶段, 浸出速率可能受一种因素控制, 也可能受多种因素 共同控制。下文着重分析时间、温度、压力, 浸出 液成分 $/ \mathrm{pH}$, 以及样品辐照损伤的影响。

\section{1 浸出时间和温度}

一般来说, 元素的浸出率会随着浸出时间的延 长而持续下降直至趋于稳定, 这与固化体-溶液界 面水化层和第二相的形成有关。如固化 $\mathrm{Ce}$ 离子的 钙钛锆石玻璃陶瓷中 $\mathrm{Ce}$ 元素浸出率在 1 3 d 内快速 降低, $7 \mathrm{~d}$ 后稳定在 $10^{-6}$ 数量级, 后期变化不大 ${ }^{[25]}$; 如无定形锆石的溶解开始遵从一级动力学, 随着时 间的延长溶解逐渐减缓 ${ }^{[18]}$ 。

温度对浸出速率的影响可用 Arrhenius 公式解 释 ${ }^{[26]}: \ln Q=\ln k-E_{\mathrm{a}} / R T$ ( $Q$ 为反应常数; $E_{\mathrm{a}}$ 为扩散活 化能; $k$ 为指前因数; $R$ 等于 $8.3143 \mathrm{~J} /(\mathrm{mol} \cdot \mathrm{K}) ; T$ 为绝 对温度)。随着温度的升高, 各个基元反应速率(包括 正向和逆向反应)变大。

玻璃固化体浸出率受温度的影响很大, 嗍硅酸 盐玻璃在 $120{ }^{\circ} \mathrm{C}$ 浸出率是 $25{ }^{\circ} \mathrm{C}$ 时的 1000 倍 ${ }^{[3]}$ 。这 是由于两种温度下反应机理不同, 低温时 $\left(<70{ }^{\circ} \mathrm{C}\right)$ 受离子交换反应控制, 高温度时受网络溶解反应控 制 ${ }^{[3]}$ 。陶瓷固化体的 $E_{\mathrm{a}}$ 较低, 受温度的影响较小, 独 居石更为独特, 浸出率随温度的升高而下降 ${ }^{[27]}$ 。此 外, 温度对固化体内损伤缺陷的恢复也有影响, 进
而影响浸出率。理论上温度升高, 缺陷变少, 如对因 自辐照而蜕晶化的矿物退火处理后发现了蜕晶部分 的重结晶 ${ }^{[28]}$ 。需要指出的是, 温度对同一样品中不 同元素的影响不同，如滕元成等 ${ }^{[29]}$ 在研究合成榍石 时发现, 温度对 $\mathrm{Ti}^{4+}$ 浸出影响较小; 对 $\mathrm{Ca}^{2+}$ 浸出前 期有一定影响, 但对后期(42d)影响较小。

\section{2 浸出压力}

压力影响化学稳定性的研究很少, 表 5 给出了 天然锆石水热蚀变受压力的影响。总的来说，压力 促进了锆石中 $\mathrm{Pb} 、 \mathrm{U}$ 的浸出, 使得锆石基体中 $\mathrm{Pb}$ 、 $\mathrm{U}$ 含量变少。

\section{3 浸出液成分}

浸出液模拟的是不同处置库中的地下水成分, 目前研究的浸出液有去离子水，硅酸盐溶液和来自 岩浆期后的、变质的、交代的流体氯化盐溶液，以 及后来扩展的强酸、强碱溶液等。在进行固化体化 学稳定性的评估时, 需要考虑地下水与纯水的差 异。Hayward 等 ${ }^{[18]}$ 根据加拿大地盾不同深度 $(300$ 、 $500 、 700 \mathrm{~m})$ 地下水的成分不同，研究了不同离子强 度的盐水对榍石浸出的影响。结果显示三种 $\mathrm{Na}-\mathrm{Ca}-\mathrm{Cl}$ 盐水中影响相似, 但是与去离子水的差别很大。 Rizvanova 等 ${ }^{[31]}$ 研究了氯化物、碳酸盐对天然锆石水 热蚀变的影响, 发现在纯水中锆石的溶解度最小, 在 碳酸盐及碱溶液中都有明显的增长; 但是在 $1 \mathrm{~mol} / \mathrm{L}$ $\mathrm{NaHCO}_{3}$ 和 $0.1 \mathrm{~mol} / \mathrm{L} \mathrm{Na}_{2} \mathrm{CO}_{3}$ 中的差异很小 ${ }^{206} \mathrm{~Pb}$ 分 别为 $5.14 \times 10^{-7}$ 和 $5.82 \times 10^{-7} \mathrm{~mol} / \mathrm{g}$ )。Berger 等 ${ }^{[21]}$ 同 样证明溶液中氟、碳酸盐、氢氧化物的存在将促进 独居石中 REE 的浸出, 使其明显高于纯水。这就提 醒我们需要有针对性地根据特定的地质条件进行浸 出实验。

固体的溶解以及其中元素的浸出不仅跟溶液成 分有关，还受溶液中关键组分的浓度的影响。 Rizvanova 等 ${ }^{[31]}$ 将 $\mathrm{Na}_{2} \mathrm{CO}_{3}$ 浓度从 $0.1 \mathrm{~mol} / \mathrm{L}$ 升高到 $1 \mathrm{~mol} / \mathrm{L}$ 时 $\left(\mathrm{pH}\right.$ 基本一致), 发现锆石中的 ${ }^{206} \mathrm{~Pb}$ 从

表 5 压力对锆石蚀变的影响

Table 5 Effect of pressure on alteration upon zircon

\begin{tabular}{|c|c|c|c|c|c|}
\hline Ref. & Liquid & Temperature $/{ }^{\circ} \mathrm{C}$ & Pressure $/ \mathrm{Pa}$ & The influence mode of pressure on zircon & Conclusion \\
\hline \multirow[b]{2}{*}[30]{} & \multirow[b]{2}{*}{$\begin{array}{l}0.1 \mathrm{~mol} / \mathrm{L} \\
\mathrm{HCl}\end{array}$} & \multirow[b]{2}{*}{400} & $0-1.5 \times 10^{8}$ & No significant change of IR & \multirow{2}{*}{$\begin{array}{l}\text { Special } \mathrm{SiO}_{2} \text { structure } \\
\text { appears under } 2.5 \mathrm{kbar}\end{array}$} \\
\hline & & & $2.5 \times 10^{8}$ & $\begin{array}{l}\text { The IR peak at } 1050 \mathrm{~cm}^{-1} \text { splitting into } \\
1049 \mathrm{~cm}^{-1} \text { and } 1087 \mathrm{~cm}^{-1}\end{array}$ & \\
\hline \multirow{5}{*}[31]{} & \multirow{5}{*}{$\begin{array}{l}2 \mathrm{~mol} / \mathrm{L} \\
\mathrm{Na}_{2} \mathrm{CO}_{3}\end{array}$} & & 0 & $33.1 \times 10^{-7} \mathrm{~mol} / \mathrm{g}{ }^{206} \mathrm{~Pb}, 101 \times 10^{-7} \mathrm{~mol} / \mathrm{g}^{238} \mathrm{U}$ & \multirow{3}{*}{$\begin{array}{l}\text { Pressure may accelerate the } \\
\text { penetration of liquid into } \\
\text { zircon matrix at } 400{ }^{\circ} \mathrm{C}\end{array}$} \\
\hline & & 400 & $1 \times 10^{8}$ & $11.4 \times 10^{-7} \mathrm{~mol} / \mathrm{g}{ }^{206} \mathrm{~Pb}, 19.2 \times 10^{-7} \mathrm{~mol} / \mathrm{g}^{238} \mathrm{U}$ & \\
\hline & & & $5 \times 10^{8}$ & $0.18 \times 10^{-7} \mathrm{~mol} / \mathrm{g}^{206} \mathrm{~Pb}, 82.0 \times 10^{-7} \mathrm{~mol} / \mathrm{g}^{238} \mathrm{U}$ & \\
\hline & & \multirow[b]{2}{*}{800} & $1 \times 10^{8}$ & $0.67 \times 10^{-7} \mathrm{~mol} / \mathrm{g}^{206} \mathrm{~Pb}, 126.0 \times 10^{-7} \mathrm{~mol} / \mathrm{g}^{238} \mathrm{U}$ & \multirow{2}{*}{$\begin{array}{l}\text { Little variation of } \mathrm{U} \text { in } \\
\text { zircon, but significant } \\
\text { variation for } \mathrm{Pb}\end{array}$} \\
\hline & & & $5 \times 10^{8}$ & $0.68 \times 10^{-7} \mathrm{~mol} / \mathrm{g}^{206} \mathrm{~Pb}, 92.4 \times 10^{-7} \mathrm{~mol} / \mathrm{g}^{238} \mathrm{U}$ & \\
\hline
\end{tabular}


$5.82 \times 10^{-7} \mathrm{~mol} / \mathrm{g}$ 降低到 $2.79 \times 10^{-7} \mathrm{~mol} / \mathrm{g}$ 。 Tribet 等 ${ }^{[32]}$ 发现钻钛锆石浸出液中的元素浓度主要由 $\mathrm{Ti}$ 和 $\mathrm{Zr}$ 的氢氧化物溶解度 (分别为 $4 \times 10^{-8}$ 和 $10^{-8} \mathrm{~mol} \cdot \mathrm{L}^{-1}$ ) 控 制, 而 $\mathrm{Ti}(\mathrm{OH})_{4}$ 的溶解度又影响 $\mathrm{Nd}$ 的浸出。Kohler 等 ${ }^{[16]}$ 对天然磷灰石的研究发现, 浸出液中的 $\mathrm{REE} / \mathrm{Ca}$ 比值与其在磷灰石中的不一致, 这是由于 $\mathrm{REE}$ 不饱和时比 $\mathrm{Ca}$ 更容易发生水化反应 $\left(K_{\mathrm{sp}} \approx\right.$ $\left.10^{-24.5 \pm 0.5}\right)$ 而优先析出; REEs 过饱和时形成磷稀土矿 (Rhabdophane, $\mathrm{REE}\left(\mathrm{PO}_{4}\right) \cdot n \mathrm{H}_{2} \mathrm{O}$ ) 而沉淀, 并附着在固 体表面, 从而使溶液中 $\mathrm{REE} / \mathrm{Ca}$ 比值减小。反应容器 材料对浸出也有影响, 这来自于背景电解质的改变, 如 Icenhower 等 ${ }^{[17]}$ 在浸出液中测得了从 Teflon 反应 釜析出的氟离子 $\left(10^{-4}\right)$ 。

\section{4 浸出液 $\mathbf{p H}$}

在固化体与浸出液的反应中, 浸出液的 $\mathrm{pH}$ 会 发生变化, 这种变化主要来自于: (1)硅酸根等基团 的溶解形成的硅酸 $\mathrm{H}_{3} \mathrm{SiO}_{4}$ 所导致的 $\mathrm{pH}$ 降低; (2)固 化体中低价金属(如 $\mathrm{Cs}^{+} 、 \mathrm{Sr}^{2+}$ 等) 与水中 $\mathrm{H}_{3} \mathrm{O}^{+}$发生离 子交换反应导致的 $\mathrm{pH}$ 升高。反过来, 浸出液 $\mathrm{pH}$ 又 影响核素的浸出率, 一般中性环境最小, 碱性环境 次之, 酸性环境最大。Poml 等 ${ }^{[33]}$ 发现掺铈、钚的钙 钛锆石在 $1 \mathrm{~mol} / \mathrm{L} \mathrm{HCl}$ 溶液中(属于强酸) 浸出率是在
$\mathrm{NaCl} 、 \mathrm{NaOH} 、 \mathrm{NH}_{3}$ 、纯水中的 10 倍。而相同条件 下温度引起的变化很小。由烧绿石 ${ }^{[34]}$ (式 4) 和钙钛锆 石 ${ }^{[33]}$ (式 5)的溶解一沉淀反应方程式可知, $\mathrm{H}^{+}$浓度越 高, 矿物越容易溶解。

$$
\begin{gathered}
\mathrm{A}_{2} \mathrm{Ti}_{2} \mathrm{O}_{7}+6 \mathrm{H}^{+} \rightarrow 2 \mathrm{TiO}_{2} \downarrow+2 \mathrm{~A}_{\mathrm{aq}}{ }^{+}+3 \mathrm{H}_{2} \mathrm{O} \\
\left(\mathrm{Ca}_{1-x} \mathrm{Ce}_{x}\right) \mathrm{Zr}\left(\mathrm{Ti}_{2-y} \mathrm{Al}_{y}\right) \mathrm{O}_{7}+\mathrm{H}^{+} \rightarrow \mathrm{ZrO}_{2} \downarrow+ \\
(2-y) \mathrm{TiO}_{2} \downarrow+(1-x) \mathrm{Ca}^{2+}{ }_{\text {aq }}+x \mathrm{Ce}^{4+}{ }_{\text {aq }}+ \\
y \mathrm{Al}^{3+}{ }_{\text {aq }}+(3+y) \mathrm{H}_{2} \mathrm{O}
\end{gathered}
$$

\section{5 损伤缺陷}

高放固化体中含约 $10 \mathrm{wt} \%$ 以上的 HLW(含有 $\alpha$ 衰变体 $\mathrm{U} 、 \mathrm{~Np} 、 \mathrm{Pu} 、 \mathrm{Am} 、 \mathrm{Cm}$ 和 $\beta$ 衰变体 ${ }^{137} \mathrm{Cs}$ 、 ${ }^{90} \mathrm{Sr}$ )，始终受到自身放射性核素的辐照，使得不同 处置阶段的辐照损伤程度不同。目前大多数实验室 不具备放射化学操作条件, 因而固化体抗浸出性能 受辐照损伤的影响研究较少。这种影响可能来自三 个方面: 一是辐照会导致材料原子层次上的变化, 进而影响物理和化学性能; 二是辐照使固化体产生 微裂隙, 改变了表面及内部形貌; 三是浸出液受辐 照产生 $\mathrm{H}^{*}, \mathrm{NO}_{3} \cdot \mathrm{H}_{2} \mathrm{O}_{2}, \mathrm{HNO}_{3}$ 等辐解产物, 改变了 固化体界面环境 ${ }^{[17,20]}$ 。表 6 总结了含有辐照损伤材

\begin{tabular}{|c|c|c|c|}
\hline Ref. & Radiated material & Effect of radiation damage on leaching rate & Conclusion \\
\hline \multirow[t]{2}{*}{ [35] } & \multirow{2}{*}{$\begin{array}{l}\text { Incorporate radionuclides } \\
\text { with short half-lives, } \\
{ }^{238} \mathrm{Pu}(87.7 \text { years }) \text { and } \\
{ }^{244} \mathrm{Cm}(17.6 \text { years })\end{array}$} & $\begin{array}{l}\text { The leaching rates }\left(\times 10^{-3}, \mathrm{~g} /\left(\mathrm{m}^{2} \cdot \mathrm{d}\right) \text { of synthetic rock containing } 1 \mathrm{wt} \% \mathrm{Cm} \text { is }\right. \\
100 \text { times of that containing } 4 \times 10^{-4} \mathrm{wt} \% \mathrm{Cm}\end{array}$ & Effective \\
\hline & & $\begin{array}{l}\text { Compared the samples containing }{ }^{238} \mathrm{Pu} \text { (regard as irradiated damage) with } \\
\text { samples containing }{ }^{239} \mathrm{Pu}\left(2.41 \times 10^{4} \text { years half-life, regard as no-radiation }\right. \\
\text { damage), the leaching rates of Pyrochlore }\left(12.35 \mathrm{wt} \% \mathrm{PuO}_{2}, 20.82 \mathrm{wt} \% \mathrm{UO}_{2}\right) \text {, } \\
\text { Pyrochlore-Rich Baseline }\left(1.88 \mathrm{wt} \% \mathrm{PuO}_{2}, 23.67 \mathrm{wt} \% \mathrm{UO}_{2}\right), \mathrm{Zirconolite} \\
\left(7.39 \mathrm{wt} \% \mathrm{PuO}_{2}\right) \text { appear with approximately equal }\left(3.2 \times 10^{-4} \mathrm{~g} /\left(\mathrm{m}^{2} \cdot \mathrm{d}\right)\right)\end{array}$ & $\begin{array}{l}\text { Little } \\
\text { effective }\end{array}$ \\
\hline$[36]$ & $\begin{array}{l}\text { Natural minerals con- } \\
\text { taining radionuclides }\end{array}$ & $\begin{array}{l}\text { The leaching rate of } \mathrm{Zr} \text { from irradiated zircon }\left(1.8 \times 10^{-2} \mathrm{~g} /\left(\mathrm{m}^{2} \cdot \mathrm{d}\right) \text { is } 10-100\right. \\
\text { times than that of undamaged zircon }\end{array}$ & Effective \\
\hline [37] & $\begin{array}{l}\text { Accelerator ion } \\
\text { implantation }\end{array}$ & $\begin{array}{l}\text { The dissolution rate of pyrochlore bombarded by heavy ion is } 50 \text { times higher } \\
\text { than that of non-bombarded }\end{array}$ & Effective \\
\hline
\end{tabular}
料的化学稳定性研究成果。

表 6 含辐照损伤的陶瓷化学稳定性研究总结

Table 6 Chemical durability study of ceramic containing radiation damage

Geisler 等 ${ }^{[38]}$ 研究了 16 个不同辐照损伤的锆石 的水化层厚度与蜕晶化程度 $p$ 的关系(水热条件为 $2 \mathrm{~mol} / \mathrm{L} \mathrm{CaCl}_{2}$ 溶液, $600{ }^{\circ} \mathrm{C}, 100 \mathrm{MPa}$ ) (图 1)。图 1 存在两个拐点, 分别在 $p=0.3$ 和 $p=0.7$ 左右。对于 $0.3<p<0.7$, 曲线可表示为 $x^{2} \propto(p-0.3)^{2}$; 对于 $p>0.7$, 曲线可表示为 $x^{2} \propto(p-0.3)^{2}+(p-0.7)^{2}$ 。Geisler 使用渗 透(Percolation) 理论 ${ }^{[39-40]}$ 解释这种辐照损伤引起的 浸出行为的差别, 认为 $p=0.3$ 对应蜕晶化结构刚刚 连接成簇; 而 $p=0.7$ 对应蜕晶化引起的结构畸变已 经允许浸出液的渗入。根据辐照损伤预测浸出率, 这对 HLW 固化体长期化学稳定性的评估有着重要 的意义。

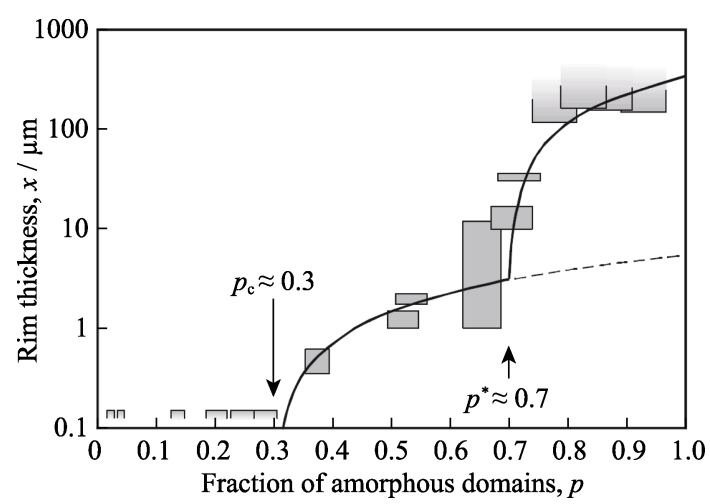

图 1 水化层厚度与非晶化程度 $p$ 的关系 ${ }^{[38]}$

Fig. 1 Relationship between hydration layer thickness and amorphous degree $p^{[38]}$ 
Tribet 等 ${ }^{[32]}$ 研究了 $\mathrm{He}^{2+}$ 、质子束辐照对钙钛锆 石溶解行为的影响, 结果显示: (1) $\mathrm{He}^{2+}$ 、质子束只辐 照水使水辐解, 辐解产物加速了钻钛锆石的溶解, 缩短了达到溶解平衡的时间; 钙钛锆石在辐解水中 的反应遵从一级动力学, 随后也受热力学的影响。 (2) $\mathrm{He}^{2+}$ 、质子束使钙钛锆石产生损伤并使水辐解, 此条件下反应机理只有一级动力学, 并且 $\mathrm{Ti} 、 \mathrm{Zr}$ 、 $\mathrm{Nd}$ 的浸出率也低于前者。Tribet 认为高能量是达到 热力学状态的必要条件, $\mathrm{Ti}$ 和 $\mathrm{Zr}$ 只有在高能下(第一 种情况)接近其氢氧化物溶解度限值 $\left(E_{\mathrm{deposited}} \approx 900 \mathrm{~J}\right)$; 固体受辐照将导致内部及界面处元素的离子化, 进 而改变化学平衡。

\section{4 可能的浸出机制}

目前可见的陶瓷固化体的浸出机制有溶解一沉 淀、扩散、离子交换几种。这些机制只是在观察少 量浸出实验后, 发现其符合某种物理假设而提出, 并没有得到大量的实验验证。

\section{1 溶解一沉淀 (Dissolution-Reprecipitation) 模型}

前文(2.3 节)提到，固化体表面可能发生水化反 应而溶解, 一些水解产物 $\mathrm{A}_{\mathrm{aq}}{ }^{+}$以胶体形式附着于固 体表面。如 $\mathrm{Pb}$ 在碱性溶液 $\left(\mathrm{NaOH} 、 \mathrm{NaHCO}_{3} 、 \mathrm{Na}_{2} \mathrm{CO}_{3}\right)$ 中溶解度很低, 一旦浸出便结合 $\mathrm{SiO}_{3}{ }^{2+}$ 生成胶体 ${ }^{[31]}$, 抑制进一步的溶解。同时, 随着固-液反应的进行, 另一些达到饱和浓度的离子将发生沉淀、絮凝、沉 积, 即所谓的溶解一沉淀浸出模型。Hayward 等 ${ }^{[18]}$ 使用溶解一沉淀机制解释了锆石的浸出, 认为无定 形锆石的溶解遵从一级动力学, 实验得到的溶解活 化能为 $12.8 \mathrm{~kJ} / \mathrm{mol}$ 。Trocellier 等 ${ }^{[41]}$ 也认为锆石属于 溶解一沉淀机制, 此过程伴随 $\mathrm{SiO}_{2}$ 的选择性释放和 表面的水解, 随之在表面形成 $\mathrm{Zr} / \mathrm{Si}$ 原子比大于 1 的 均匀水化层。Trocellier 等 ${ }^{[33]}$ 得出钲钛锆石的活化能 是 $8 \sim 20 \mathrm{~kJ} / \mathrm{mol}$, 认为晶态钙钛锆石界面上 $\mathrm{ZrO}_{2}$ 和 $\mathrm{TiO}_{2}$ 的生成也可用耦合界面的溶解一沉淀机制解释, 原始钙钛锆石一致溶解, 反应前沿向内移动, 由于 溶解度差异将逐步沉淀出 $\mathrm{ZrO}_{2}$ 和 $\mathrm{TiO}_{2}$ 。同时 $\mathrm{ZrO}_{2}$ 和 $\mathrm{TiO}_{2}$ 的沉淀促进钙钛锆石的的溶解。

\section{2 扩散控制(Diffusion-Control)模型}

Geisler 等 ${ }^{[42]}$ 报道了水热条件会使因辐照损伤 而蜕晶的锆石出现一定程度的晶格恢复 ${ }^{[43]}$, 甚至重 结晶。溶液-沉淀得到的锆石一般裹有杂相(图 2(B)中 红色区域), 且会造成其中 $\mathrm{Pb}$ 的丢失(由溶解度差异 导致)。而这种蚀变的锆石含有 $\mathrm{Pb}$, 不含杂相, 说明 这种水热蚀变更可能是由离子扩散反应控制, 而不

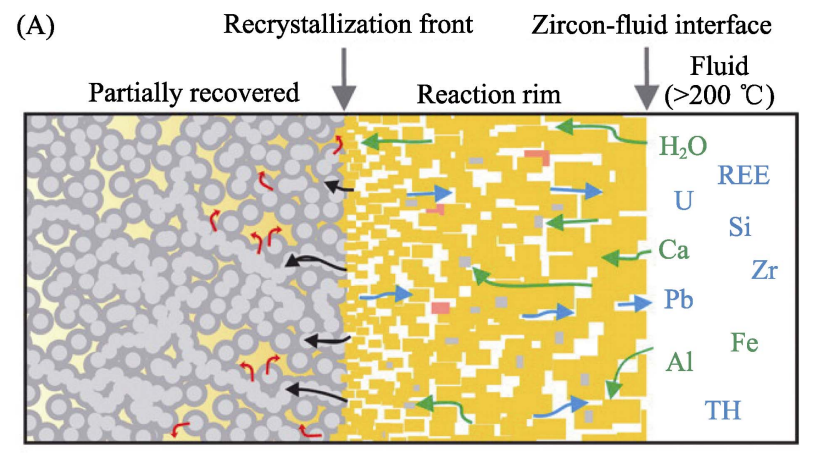

Structural disorder in crystalline zircon domains

\begin{tabular}{|lc|}
\hline Highly disordered (gradual) & $\begin{array}{c}\text { Little disordered } \\
\text { (temerature dependent) }\end{array}$ \\
\hline
\end{tabular}
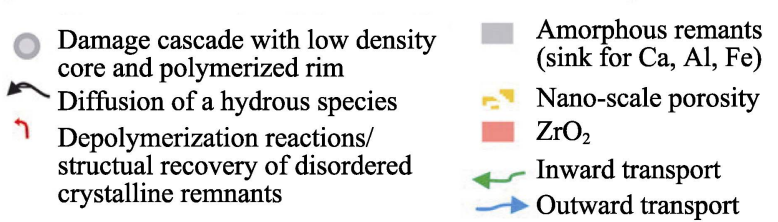

(B) Dissolution-reprecipitation front $\quad$ Zircon-fluid interface
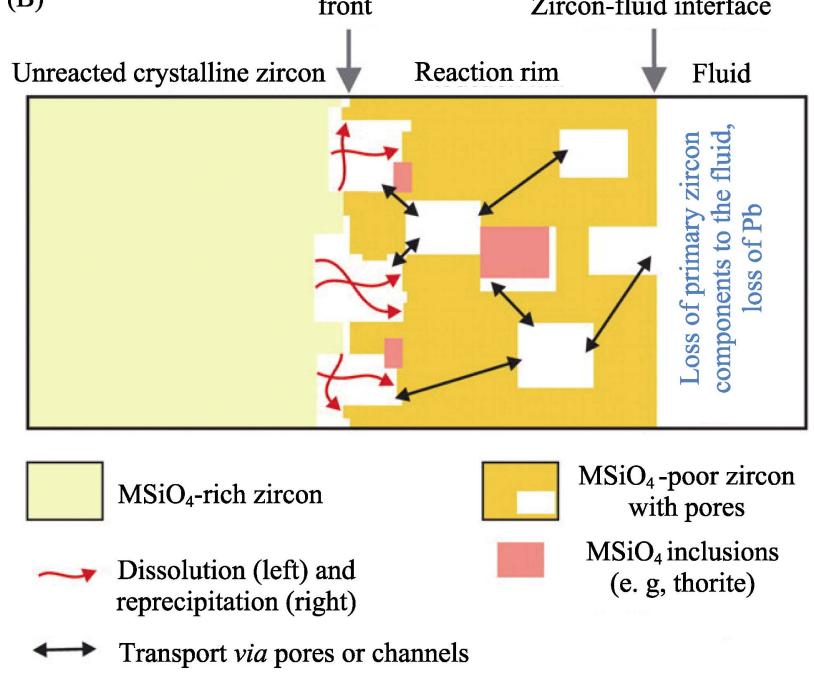

图 2 (A)扩散反应机制和(B)溶解一再沉淀耦合的原理图 ${ }^{[44]}$

Fig. 2 Schematic outline of the diffusion-reaction (A) and coupled dissolution-reprecipitation mechanism $(B)^{[44]}$

是溶解一沉淀机制。重结晶的出现使此区域的原子体 积降低( 18\%), 产生内部结构应力, 并以产生裂纹 的形式释放。而裂纹及这些低原子密度的区域有利 于离子扩散。在固-液界面与无反应区之间, 重结晶 程度、阳离子 $\left(\mathrm{Ca}^{2+} 、 \mathrm{Al}^{3+} 、 \mathrm{Fe}^{3+}\right.$, 由 $\mathrm{IMP}$ 与 $\mathrm{EM}$ 证 明)、裂纹等都存在着明显的梯度。伴随着 $\mathrm{H}^{+}$或分 子水的扩散重结晶前沿向里移动。

Geisler 总结了溶解一沉淀、扩散控制模型的区 别 ${ }^{[44]}$ (图 2)。从图 2(A) 可以看出, 扩散引起较微观的 变化, 如出现的孔、第二相为纳米级, 结构恢复与温 度有关, 所含同位素显示有母体的记忆效应。从图 2(B) 可以看出, 溶解一沉淀机制对应较宏观的变化, 如 出现的孔为微米级, 第二相以包裹物的形式出现, 同位素比值变化较大(可能是完全再平衡造成的)。 


\section{3 离子交换}

人工合成的铅-钒-碘-磷灰石 [Lead vanadoiodoapatite, $\left.\mathrm{Pb}_{5}\left(\mathrm{VO}_{4}\right)_{3} \mathrm{I}\right]$ 在水中的浸出反应有:

溶解, $\left[\mathrm{Pb}_{5}\left(\mathrm{VO}_{4}\right)^{3} \mathrm{I}\right] \rightarrow 5 \mathrm{~Pb}_{2}++3\left(\mathrm{VO}_{4}\right)^{-3}+\mathrm{I}^{-1}$

$\mathrm{Pb}$ 沉淀, $\mathrm{Pb}^{2+}+2 \mathrm{OH}^{-1} \rightarrow \mathrm{Pb}(\mathrm{OH})_{2}$

$\mathrm{I}$ 取代, $(\mathrm{VO} 4)^{-3} \mathrm{I}^{-1}+\mathrm{OH}^{-1} \rightarrow\left(\mathrm{VO}_{4}\right)^{-3} \mathrm{OH}^{-1}+\mathrm{I}^{-1}$

Zhang 等 ${ }^{[45]}$ 发现其浸出的 $\mathrm{I} / \mathrm{V}$ 摩尔比与基体 $\mathrm{I} / \mathrm{V}=1 / 3$ 不符, $\mathrm{I}$ 高于 $\mathrm{V}$ 。使用 Raman 光谱(对 $\mathrm{OH}$ 和 $\mathrm{H}_{2} \mathrm{O}$ 极为敏感)证实了磷灰石晶体中 $\mathrm{OH} 、 \mathrm{HCO}_{3}$ 的 存在, 说明 $\mathrm{OH}$ 与 $\mathrm{I}$ 或少量 $\mathrm{V}$ 可能发生了取代反应 (式 8)。目前还不确定是否有以下反应(式 9)发生:

$$
\left(\mathrm{VO}_{4}\right)^{-3} \mathrm{I}^{-1}+\mathrm{HCO}_{3}^{-1} \rightarrow\left(\mathrm{VO}_{4}\right)^{-3} \mathrm{HCO}_{3}^{-1}+\mathrm{I}^{-1}
$$

目前基于扩散控制的离子交换反应还有烧绿石 水热蚀变, Geisler 等 ${ }^{[46]}$ 报道了天然烧绿石晶体的由 界面控制的准晶置换反应。

\section{4 模型的局限性}

在真实的地质环境中, 固化体的浸出行为非常 复杂, 很难找到一种通用的浸出模型来描述所有的 浸出行为: (1)在蜕晶化锆石的研究中发现水(更可能 是氢)降低了重结晶活化能 ${ }^{[30,44,47]}$, 即在干燥条件下 $700{ }^{\circ} \mathrm{C}$ 开始重结晶, 而在水热环境下 $200{ }^{\circ} \mathrm{C}$ 时即可 出现。溶解一沉淀和扩散控制模型都不能准确地描述 低温下水对重结晶的 “催化” 作用, 以及氢 $\left(\mathrm{H}^{+} / \mathrm{H}_{3} \mathrm{O}^{+}\right)$ 的存在为何能大大降低严重损伤锆石的成核生长温 度。(2)扩散控制模型认为氢进入锆石的位置与缺陷 有关, 却不能解释为何榍石水热反应中有氢的进入, 却没有发现重结晶。

\section{5 总结及展望}

目前化学稳定性的研究方法基本成熟, 可以满 足各类样品在各种条件下的操作参照。陶瓷有序度 高, 可以从微观级别进行表征, 这对了解固化体的 浸出行为、化学稳定性的评价方法至关重要。陶瓷 固化体化学稳定性的研究还需加强:

1) 陶瓷固化体的浸出机理与评价方法还处于 起步阶段。较多关于固化体合成、耐辐照性能的研 究, 而化学稳定性只作为辅助研究, 目前还不能确 定哪种陶瓷固化体的化学稳定性更优。

2) 认识固化体在原子尺度上的变化是理解浸 出行为及其机理的关键。目前有耐辐照性能与结构 的关系研究 ${ }^{[48]}$, 但是化学稳定性还停留在浸出率等 宏观性质方面。对放射性核素在固化体中的赋存状 态、迁移模式、价态变化的研究较少, 因此难以给 出材料结构与抗浸出性能之间的关系。一些先进技 术(如 EXAFS、XRF、XPS、同位素示踪)值得尝试。
3) 陶瓷固化体化学稳定性的主要研究对象是 不含放射性核素的模拟样品, 缺少放射性核素衰变 产生的损伤缺陷，这与真实的固化体存在着很大的 差别。研究掺入放射性核素或含辐照损伤的天然矿 物可为辐照损伤对化学稳定性的研究提供帮助。

4) 研究浸出过程中是否有离子进入固化体, 了解可能的分布、结构形态将是对固化体化学稳定 性研究的有力补充。

5) 目前研究的浸出行为的影响因素有温度、浸 出液成分、 $\mathrm{pH}$ 等。而辐照效应、气体环境、压强、 水流、微生物、容器腐蚀产物、回填材料等的研究 较少, 尤其是接近处置库条件的浸出液-温度-辐射压力_气流等多种因素耦合作用下的研究很是缺乏。

6) 国内张华等 ${ }^{[11]}$ 根据北山地下水成分丰富了 浸出液类别、根据深地层缺氧现状考虑了气氛，建 立了使用短期试验模拟长期处置情景的试验方法。 但是陶瓷固化体只有短期化学稳定性得到了研究, 长期化学稳定性同样缺乏。

\section{参考文献:}

[1] WANG J, SU R, CHEN W M, et al. Deep geological disposal of high-level radioactive wastes in China. Chinese Journal of Rock Mechanics \& Engineering, 2006, 25(4): 649-658.

[2] EWING R C. Long-term storage of spent nuclear fuel. Nature Materials, 2015, 14(3): 252-257.

[3] 谭宏斌, 李玉香. 放射性废物固化方法综述. 云南环境科学, 2004, 23(4): 1-3.

[4] CHEN F Y, JIE W Q, DELBERT E D. Corrosion property of iron phosphate simulated HLW melts. Journal of Inorganic Materials, 2000, 15(4): 653-659.

[5] EWING R C. Nuclear waste forms for actinides. Proc. Nat. Acad. Sci., 1999, 96(7): 3432-3439.

[6] WANG L L, XIE H, CHEN Q Y, et al. Synthesis and charaterization of thorium-doped $\mathrm{Nd}_{2} \mathrm{Zr}_{2} \mathrm{O}_{7}$ pyrochlore. Journal of Inorganic Materials, 2015, 30(1): 81-86.

[7] DONALD I W, METCALFE B L, TAYLOR R N J. The immobilization of high level radioactive wastes using ceramics and glasses. Journal of Materials Science, 1997, 32(22): 5851-5887.

[8] 何涌. 高放废液玻璃固化体和矿物固化体性质的比较. 辐射防 护, 2001, 21(1): 43-47.

[9] HAYWARD P J, CECCHETTO E V. Development of sphene-based glass ceramics tailored for canadian waste disposal conditions. Mater. Res. Soc. Symp. Proc., 1981, 6: 91-97.

[10] 盛嘉伟, 罗上庚, 汤宝龙. 高放废液的玻璃固化及固化体的浸 出行为与发展情况. 硅酸盐学报, 1997, 25(1): 83-88.

[11] 张华. 高放固化体处置条件下的浸出和模型研究. 北京：中国 原子能科学研究院博士学位论文, 2004.

[12] MENDEL J E. Nuclear Waste Materials Characterization Center. Topp S V, Semiannual progress report, PNL-5683 America, 1985: $1-54$.

[13] C1285-02, Standard test methods for determining chemical durability of nuclear, hazardous, and mixed waste glasses and multiphase glass ceramics: The product consistency test (PCT).

[14] 吴萍萍, 张骋, 徐海芳, 等. 玻璃固化体抗浸蚀性能实验研究进 
展. 现代技术陶瓷, 2010,31(3): 28-34.

[15] EJ/1186-2005, 放射性废物体和废物包的特性鉴定.

[16] KÖHLER S J, HAROUIYA N, CHAÏRAT C, et al. Experimental studies of REE fractionation during water-mineral interactions: REE release rates during apatite dissolution from $\mathrm{pH} 2.8$ to 9.2 . Chem. Geol., 2005, 222(3/4): 168-182.

[17] ICENHOWER J P, STRACHAN D M, LINDBERG M J, et al. Dissolution Kinetics of Titanate-based Ceramic Waste Forms: Results from Single-pass Flow Tests on Radiation Damaged Specimens. United States: N. p., DOI: 10.2172/15003935.

[18] HAYWARD P J, WATSON D G, MCILWAIN A K, et al. Leaching studies of sphene ceramics containing substituted radionuclides. Nuclear and Chemical Waste Management, 1986, 6(1): 71-80.

[19] GEISLER T, PIDGEON R T, KURTZ R, et al. Experimental hydrothermal alteration of partially metamict zircon. Am. Mineral., 2003, 88(10): 1496-1513.

[20] TOUlhOAT N, TOUlHOAT N, MONCOFFRE N, et al. Enhancement of zirconolite dissolution due to water radiolysis. MRS Proceedings, 2006, 985: 0985-NN09-04.

[21] BERGER A, GNOS E, JANOTS E, et al. Formation and composition of rhabdophane, bastnäsite and hydrated thorium minerals during alteration: implications for geochronology and low-temperature processes. Chem. Geol., 2008, 254(3): 238-248.

[22] 张华, 杨建文, 李宝军, 等. 富烧绿石在模拟处置条件下的浸出 行为研究. 核化学与放射化学, 2004, 26(2): 65-70.

[23] FRUGIER P, MARTIN C, RIBET I, et al. The effect of composition on the leaching of three nuclear waste glasses: R7T7, AVM and VRZ. J. Nucl. Mater., 2005, 346(2/3): 194-207.

[24] PIRLET V. Influence of the near-field conditions on the mobile concentrations of $\mathrm{Np}$ and Tc leached from vitrified HLW. MRS Proceedings, 2004, 824: CC7.5.

[25] 李鹏, 丁新更, 杨辉, 等. 钻钛锆石玻璃陶瓷体的晶化和抗浸出 性能. 硅酸盐学报, 2012, 40(2): 324-328.

[26] 盛嘉伟, 罗上庚, 汤宝龙, 等. 90-19/U 模拟高放玻璃固化体的 浸出特性评价. 核化学与放射化学, 1995, 17(1): 1-6.

[27] SHIN H Y, PARK H, YOO K. The effect of temperature on the leaching of monazite obtained from heavy mineral sands. Geosystem Engineering, 2012, 15(2): 118-122.

[28] HAWTHORNE F C, GROAT L A, RAUDSEPP M, et al. Alphadecay damage in titanite. Am. Mineral., 1991, 76(3/4): 370-396.

[29] 滕元成, 曾冲盛, 任雪潭, 等. 合成榍石的化学稳定性. 原子能 科学技术, 2010, 44(1): 14-19.

[30] GEISLER T, ZHANG M, SALJE E K H. Recrystallization of almost fully amorphous zircon under hydrothermal conditions: an infrared spectroscopic study. J. Nucl. Mater, 2003, 320(3): 280-291.

[31] RIZVANOVA N G, GAIDAMAKO I M, LEVCHENKOV O A, et al. Interaction of metamict zircon with fluids of various composition. Geochemistry International, 2007, 45(5): 465-477.

[32] TRIBET M, TOULHOAT N, MONCOFFRE N, et al. Leaching of a zirconolite ceramic waste-form under proton and $\mathrm{He}^{2+}$ irradiation. Radiochimica Acta, 2008, 96(9/10/11): 619-624.

[33] PÖML P, GEISLER T, COBOS-SABATÉ J, et al. The mechanism of the hydrothermal alteration of cerium- and plutonium-doped zirconolite. J. Nucl. Mater, 2011, 410(1): 10-23.

[34] PÖML P, MENNEKEN M, STEPHAN T, et al. Mechanism of hydrothermal alteration of natural self-irradiated and synthetic crystalline titanate-based pyrochlore. Geochim. Cosmochim. Acta, 2007, 71(13): 3311-3322.

[35] MitAMURA H, MATSUMOTO S, STEWART M W A, et al. $\alpha$-Decay damage effects in curium-doped titanate ceramic containing sodium-free high-level nuclear waste. J. Am. Ceram. Soc., 1994, 77(9): 2255-2264.

[36] EWING R C, HAAKER R F, LUTZE W. Leachability of zircon as a function of alpha dose. MRS Online Proceeding Library, 1980, 11(1): 389-397.

[37] BEGG B D, HESS N J, WEBER W J, et al. Heavy-ion irradiation effects on structures and acid dissolution of pyrochlores. J. Nucl. Mater., 2001, 288(2): 208-216.

[38] GEISLER T, TRACHENKO K, RÍOS S, et al. Impact of self-irradiation damage on the aqueous durability of zircon $\left(\mathrm{ZrSiO}_{4}\right)$ : implications for its suitability as a nuclear waste form. Journal of Physics Condensed Matter, 2003, 15(37): 1597-1605.

[39] SALJE E K H, CHROSCH J, EWING R C. Is "metamictization" of zircon a phase transition? Am. Mineral., 1999, 84(7/8): 1107-1116.

[40] TRACHENKO K, DOVE M T, SALJE E K H. Reply to comment on 'large swelling and percolation in irradiated zircon'. Journal of Physics Condensed Matter, 2003, 15(15): 6457-6471.

[41] TROCELLIER P, DELMAS R. Chemical durability of zircon. $\mathrm{Nu}$ clear Inst and Methods in Physics Research B, 2001, 181(1): 408-412.

[42] GEISLER T, PIDGEON R T, BRONSWIJK W V, et al. Transport of uranium, thorium, and lead in metamict zircon under lowtemperature hydrothermal conditions. Chem. Geol., 2002, 191(1): 141-154.

[43] GEISLER T, RASHWAN T, RAHN A A, et al. Low-temperature hydrothermal alteration of natural metamict zircons from the eastern desert, Egypt. Mineralogical Magazine, 2003, 67(3): 485-508.

[44] GEISLER T, SCHALTEGGER U, TOMASCHEK F. Re-equilibration of zircon in aqueous fluids and melts. Elements, 2007, 3(1): 43-50.

[45] ZHANG M, MADDRELL E R, ABRAITIS P K, et al. Impact of leach on lead vanado-iodoapatite $\left[\mathrm{Pb}_{5}\left(\mathrm{VO}_{4}\right)_{3} \mathrm{I}\right]$ : an infrared and Raman spectroscopic study. Materials Science \& Engineering B, 2007, 137(1): 149-155.

[46] GEISLER T, PÖML P, STEPHAN T, et al. Experimental observation of an interface-controlled pseudomorphic replacement reaction in a natural crystalline pyrochlore. Am. Mineral., 2005, 90(10): $1683-1687$.

[47] GEISLER T, ULONSKA M, SCHLEICHER H, et al. Leaching and differential recrystallization of metamict zircon under experimental hydrothermal conditions. Contrib. Mineral. Petrol., 2001, 141(1): 53-65.

[48] LIAN J, ZU X T, KUTTY K V G, et al. Ion-irradiation-induced amorphization of $\mathrm{La}_{2} \mathrm{Zr}_{2} \mathrm{O}_{7}$ pyrochlore. Phys. Rev. B, 2002, 66(66): 054108-1-5. 